\title{
ANOTHER SIMPLE PROOF OF THE QUINTUPLE PRODUCT IDENTITY
}

\author{
HEI-CHI CHAN
}

Received 14 December 2004 and in revised form 15 May 2005

We give a simple proof of the well-known quintuple product identity. The strategy of our proof is similar to a proof of Jacobi (ascribed to him by Enneper) for the triple product identity.

\section{Introduction}

The well-known quintuple product identity can be stated as follows. For $z \neq 0$ and $|q|<1$,

$$
\begin{aligned}
f(z, q): & =\prod_{n=0}^{\infty}\left(1-q^{2 n+2}\right)\left(1-z q^{2 n+1}\right)\left(1-\frac{1}{z} q^{2 n+1}\right)\left(1-z^{2} q^{4 n}\right)\left(1-\frac{1}{z^{2}} q^{4 n+4}\right) \\
& =\sum_{n=-\infty}^{\infty} q^{3 n^{2}+n}\left(z^{3 n} q^{-3 n}-z^{-3 n-1} q^{3 n+1}\right) .
\end{aligned}
$$

The quintuple identity has a long history and, as Berndt [5] points out, it is difficult to assign priority to it. It seems that a proof of the identity was first published in H. A. Schwartz's book in 1893 [19]. Watson gave a proof in 1929 in his work on the RogersRamanujan continued fractions [20]. Since then, various proofs have appeared. To name a few, Carlitz and Subbarao gave a simple proof in [8]; Andrews [2] gave a proof involving basic hypergeometric functions; Blecksmith, Brillhart, and Gerst [7] pointed out that the quintuple identity is a special case of their theorem; and Evans [11] gave a short and elegant proof by using complex function theory. For updated history up to the late $80 \mathrm{~s}$ and early 90s, see Hirschhorn [15] (in which the author also gave a beautiful generalization of the quintuple identity) and Berndt [5] (in which the author also gave a proof that ties the quintuple identity to the larger framework of the work of Ramanujan on $q$-series and theta functions; see also [1]). Since the early 90s, several authors gave different new proofs of the quintuple identity; see $[6,13,12,17]$. See also Cooper's papers $[9,10]$ for the connections between the quintuple product identity and Macdonald identities [18]. Quite recently, Kongsiriwong and Liu [16] gave an interesting proof that makes use of the cube root of unity. 


\section{Another simple proof of the quintuple product identity}

Our proof below is similar to the proof of the triple product identity by Jacobi (ascribed to him by Enneper; see the book by Hardy and Wright [14]). First, we set $f(z, q)=$ $\sum a_{n} z^{n}$. Then, by considering the symmetry of $f(z, q)$ as an infinite product, we relate all $a_{n}$ to a single coefficient $a_{0}$. All we need to do is to evaluate $a_{0}$. This is achieved by comparing $f(i, q)$ and $f\left(-q^{4}, q^{4}\right)$.

\section{Proof of the identity}

The first step of our proof is pretty standard, for example, see [16] or [4]. Set

$$
f(z, q)=\sum_{n=-\infty}^{\infty} a_{n} z^{n}
$$

From the definition of $f(z, q)$, one can show that

$$
f(z, q)=q z^{3} f\left(z q^{2}, q\right), \quad f(z, q)=-z^{2} f\left(\frac{1}{z}, q\right) .
$$

The first equality implies that for each $n$,

$$
a_{3 n}=a_{0} q^{3 n^{2}-2 n}, \quad a_{3 n+1}=a_{1} q^{3 n^{2}}, \quad a_{3 n+2}=a_{2} q^{3 n^{2}+2 n},
$$

whereas the second equality implies that $a_{2}=-a_{0}$ and $a_{1}=0$. By putting all these together, we have

$$
f(z, q)=a_{0}(q) \sum_{n=-\infty}^{\infty} q^{3 n^{2}+n}\left(z^{3 n} q^{-3 n}-z^{-3 n-1} q^{3 n+1}\right) .
$$

Comparing (2.4) to (1.1) shows that all we need to do is to prove that $a_{0}(q)=1$. Note that $a_{0}(0)=1$.

We can also write (2.4) in the following forms (which will be useful later):

$$
\begin{aligned}
f(z, q) & =a_{0}(q) \sum_{n=-\infty}^{\infty} q^{3 n^{2}-2 n}\left(z^{3 n}-\frac{1}{z^{3 n-2}}\right) \\
& =a_{0}(q) \sum_{n=-\infty}^{\infty} q^{3 n^{2}+n}\left(\left(\frac{z}{q}\right)^{3 n}-\left(\frac{q}{z}\right)^{3 n+1}\right) .
\end{aligned}
$$

To obtain (2.5a), we let $n \rightarrow n-1$ in the second sum on the right-hand side of (2.4). Equation (2.5b) is simply another way of writing (2.4).

By putting $z=i$ in (2.5a), we have, on the one hand,

$$
f(i, q)=a_{0}(q) \sum_{n=-\infty}^{\infty} q^{3 n^{2}-2 n}\left(i^{3 n}-\frac{1}{i^{3 n-2}}\right)=2 a_{0}(q) \sum_{n=-\infty}^{\infty} q^{12 n^{2}-4 n}(-1)^{n} .
$$

Note that, in the second equality, we have used the fact that

$$
i^{3 n}-\frac{1}{i^{3 n-2}}=2 \cos \frac{3 n}{2} \pi= \begin{cases}0, & \text { if } n \text { is odd } \\ 2(-1)^{n / 2}, & \text { if } n \text { is even }\end{cases}
$$


On the other hand, let us evaluate $f(i, q)$ as an infinite product:

$$
\begin{aligned}
f(i, q) & =2 \prod_{n=1}^{\infty}\left(1-q^{2 n}\right)\left(1-i q^{2 n-1}\right)\left(1+i q^{2 n-1}\right)\left(1+q^{4 n}\right)^{2} \\
& =2 \prod_{n=1}^{\infty}\left(1-q^{2 n}\right)\left(1+q^{4 n-2}\right)\left(1+q^{4 n}\right)^{2} \\
& =2 \prod_{n=1}^{\infty}\left(1-q^{2 n}\right)\left(1+q^{2 n}\right)\left(1+q^{4 n}\right) \\
& =2 \prod_{n=1}^{\infty}\left(1-q^{4 n}\right)\left(1+q^{4 n}\right) \\
& =2 \prod_{n=1}^{\infty}\left(1-q^{8 n}\right) .
\end{aligned}
$$

Note that we have used the fact that $\prod\left(1+q^{4 n-2}\right)\left(1+q^{4 n}\right)=\prod\left(1+q^{2 n}\right)$ to derive the third equality.

By putting (2.6) and (2.8) together, we arrive at

$$
\prod_{n=1}^{\infty}\left(1-q^{8 n}\right)=a_{0}(q) \sum_{n=-\infty}^{\infty} q^{12 n^{2}-4 n}(-1)^{n}
$$

Note that, at this stage, if we appeal to Euler's pentagonal number theorem (with $q$ replaced by $q^{8}$ ) [4], we have

$$
\prod_{n=1}^{\infty}\left(1-q^{8 n}\right)=\sum_{n=-\infty}^{\infty} q^{12 n^{2}-4 n}(-1)^{n}
$$

Compared with (2.9), we see that $a_{0}(q)=1$. Alternatively, we can find $a_{0}(q)$ by evaluating $f(z, q)$ in a different way.

Precisely, let us evaluate $f\left(-q^{4}, q^{4}\right)$. By $(2.5 \mathrm{~b})$, we have

$$
\begin{aligned}
f\left(-q^{4}, q^{4}\right) & =a_{0}\left(q^{4}\right) \sum_{n=-\infty}^{\infty} q^{12 n^{2}+4 n}\left((-1)^{3 n}-(-1)^{3 n+1}\right) \\
& =2 a_{0}\left(q^{4}\right) \sum_{n=-\infty}^{\infty} q^{12 n^{2}+4 n}(-1)^{n} \\
& =2 a_{0}\left(q^{4}\right) \sum_{n=-\infty}^{\infty} q^{12 n^{2}-4 n}(-1)^{n} .
\end{aligned}
$$

For the second equality, we have used the fact that $(-1)^{3 n}-(-1)^{3 n+1}=2(-1)^{n}$. For the last equality, we let $n \rightarrow-n$ in the second line.

Again, evaluating $f\left(-q^{4}, q^{4}\right)$ as an infinite product gives

$$
f\left(-q^{4}, q^{4}\right)=2 \prod_{n=1}^{\infty}\left(1-q^{8 n}\right)\left(\prod_{k=1}^{\infty}\left(1+q^{8 k}\right)\left(1-q^{16 k-8}\right)\right)^{2}=2 \prod_{n=1}^{\infty}\left(1-q^{8 n}\right) .
$$




\section{Another simple proof of the quintuple product identity}

The second equality is obtained by direct computation, similar to the derivation of (2.8). Alternatively, it follows from an identity due to Euler (e.g., see [3, page 60]) that

$$
\prod_{k=1}^{\infty}\left(1+q^{8 k}\right)\left(1-q^{16 k-8}\right)=1 .
$$

By putting together (2.11) and (2.12), we have

$$
\prod_{n=1}^{\infty}\left(1-q^{8 n}\right)=a_{0}\left(q^{4}\right) \sum_{n=-\infty}^{\infty} q^{12 n^{2}-4 n}(-1)^{n}
$$

Finally, by comparing (2.9) and (2.14), we conclude that $a_{0}(q)=a_{0}\left(q^{4}\right)$. This implies that

$$
a_{0}(q)=a_{0}\left(q^{4}\right)=a_{0}\left(q^{16}\right)=\cdots=a_{0}\left(q^{4^{k}}\right)=\cdots=a_{0}(0)=1
$$

and (1.1) is proven.

We remark that the evaluation of $f\left(-q^{4}, q^{4}\right)$ above also gives a simple proof of Euler's pentagonal number theorem.

\section{Acknowledgments}

I would like to thank Douglas Woken for his support and encouragement and Shaun Cooper for his valuable comments and encouragement. Also, I would like to thank the referees, whose comments were extremely valuable and encouraging.

\section{References}

[1] C. Adiga, B. C. Berndt, S. Bhargava, and G. N. Watson, Chapter 16 of Ramanujan's second notebook: theta-functions and q-series, Mem. Amer. Math. Soc. 53 (1985), no. 315, v+85.

[2] G. E. Andrews, Applications of basic hypergeometric functions, SIAM Rev. 16 (1974), no. 4, 441484.

[3] - q-Series: Their Development and Application in Analysis, Number Theory, Combinatorics, Physics, and Computer Algebra, CBMS Regional Conference Series in Mathematics, vol. 66, American Mathematical Society, Rhode Island, 1986.

[4] G. E. Andrews, R. Askey, and R. Roy, Special Functions, Encyclopedia of Mathematics and Its Applications, vol. 71, Cambridge University Press, Cambridge, 1999.

[5] B. C. Berndt, Ramanujan's Notebooks. Part III, Springer, New York, 1991.

[6] S. Bhargava, A simple proof of the quintuple product identity, J. Indian Math. Soc. (N.S.) 61 (1995), no. 3-4, 226-228.

[7] R. Blecksmith, J. Brillhart, and I. Gerst, New proofs for two infinite product identities, Rocky Mountain J. Math. 22 (1992), no. 3, 819-823.

[8] L. Carlitz and M. V. Subbarao, A simple proof of the quintuple product identity, Proc. Amer. Math. Soc. 32 (1972), no. 1, 42-44.

[9] S. Cooper, The Macdonald identities for $G_{2}$ and some extensions, New Zealand J. Math. 26 (1997), no. 2, 161-182.

[10] Multiplying Macdonald identities, Special Functions and Differential Equations (Madras, 1997), Allied Publishers, New Delhi, 1998, pp. 73-82.

[11] R. J. Evans, Theta function identities, J. Math. Anal. Appl. 147 (1990), no. 1, 97-121.

[12] D. Foata and G.-N. Han, Jacobi and Watson identities combinatorially revisited, Sém. Lothar. Combin. 42 (1999), no. B42o, 12 pp. 
[13] The triple, quintuple and septuple product identities revisited, Sém. Lothar. Combin. 42 (1999), Article B42o, pp 12.

[14] G. H. Hardy and E. M. Wright, An Introduction to the Theory of Numbers, 5th ed., The Clarendon Press, Oxford University Press, New York, 1979.

[15] M. D. Hirschhorn, A generalisation of the quintuple product identity, J. Austral. Math. Soc. Ser. A 44 (1988), no. 1, 42-45.

[16] S. Kongsiriwong and Z.-G. Liu, Uniform proofs of q-series-product identities, Results Math. 44 (2003), no. 3-4, 312-339.

[17] V. E. Leininger and S. C. Milne, Some new infinite families of $\eta$-function identities, Methods Appl. Anal. 6 (1999), no. 2, 225-248.

[18] I. G. Macdonald, Affine root systems and Dedekind's $\eta$-function, Invent. Math. 15 (1972), 91143.

[19] H. A. Schwarz, Formeln und Lehrsätze zum Gebrauche der Elliptischen Funktionen. Nach Vorlesungen und Aufzeichnungen des Herrn Prof. K. Weierstrass, Zweite Ausgabe, Erste Abteilung, Springer, Berlin, 1893.

[20] G. N. Watson, Theorems stated by Ramanujan (VII): Theorems on continued fractions, J. London Math. Soc. 4 (1929), 39-48.

Hei-Chi Chan: Mathematical Science Program, University of Illinois at Springfield, Springfield, IL 62703-5407, USA

E-mail address: chan.hei-chi@uis.edu 


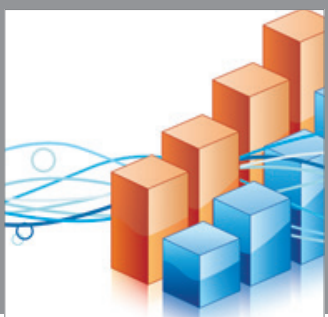

Advances in

Operations Research

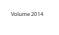

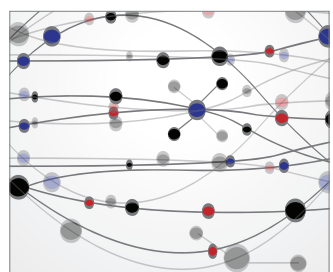

\section{The Scientific} World Journal
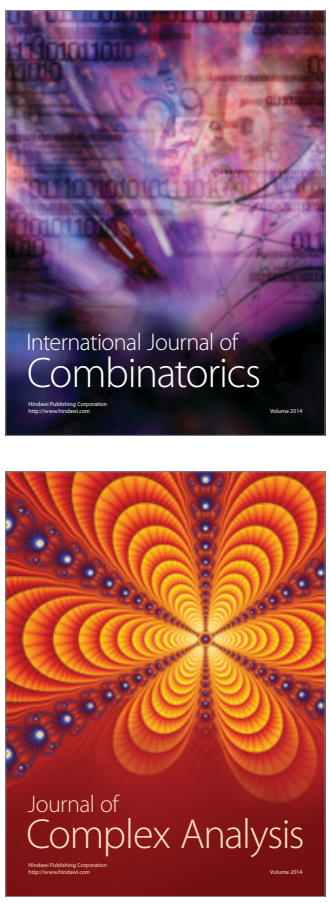

International Journal of

Mathematics and

Mathematical

Sciences
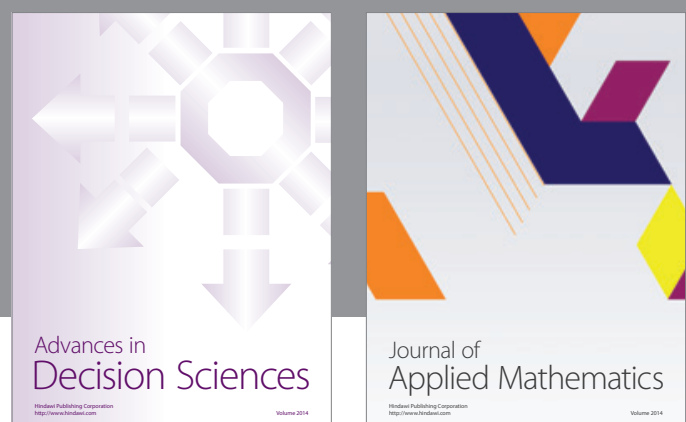

Journal of

Applied Mathematics
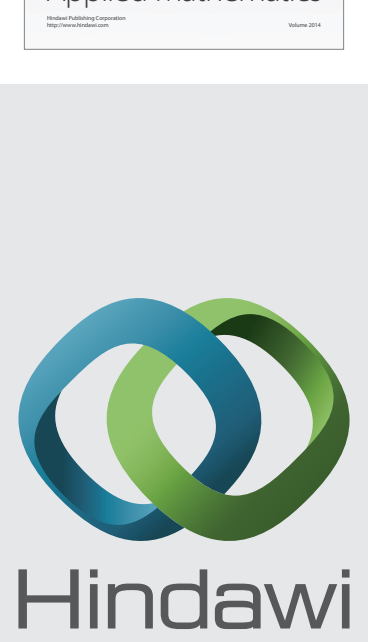

Submit your manuscripts at http://www.hindawi.com
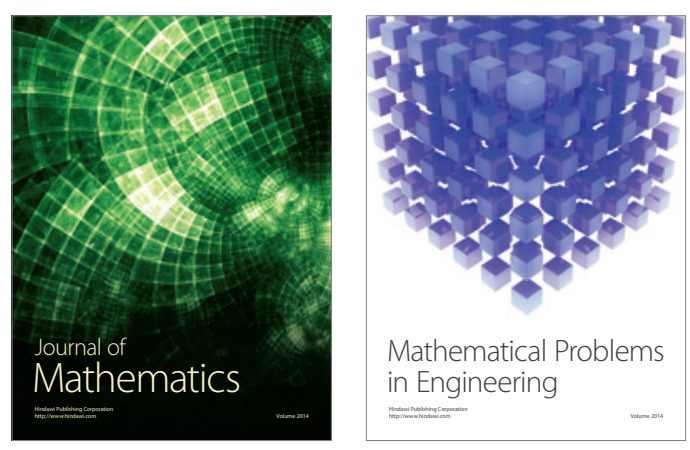

Mathematical Problems in Engineering
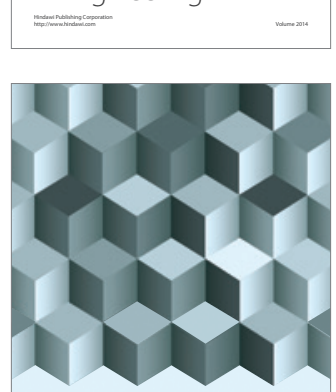

Journal of

Function Spaces
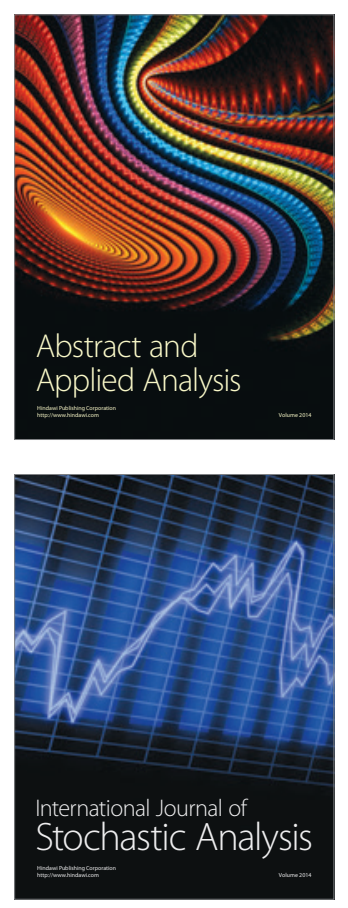

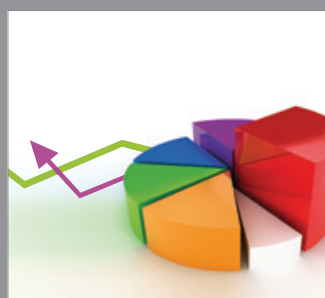

ournal of

Probability and Statistics

Promensencen
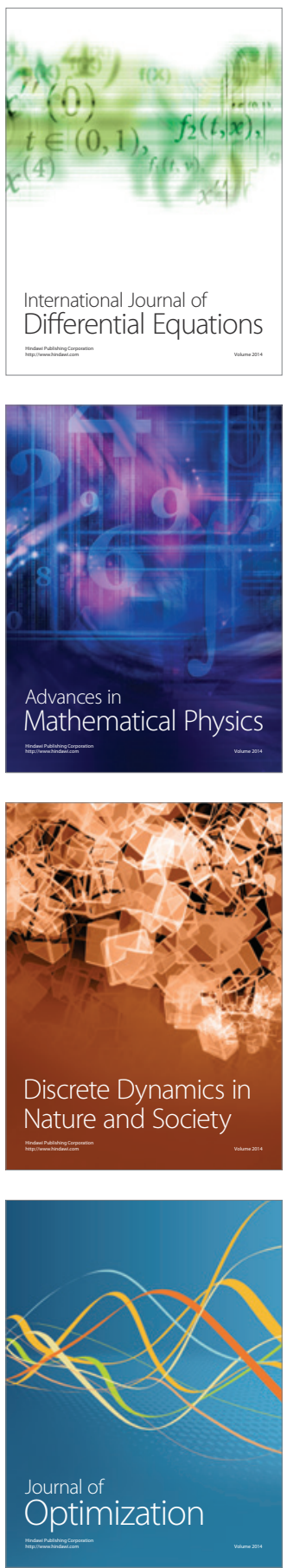